\author{
Supporting Information
}

\title{
Se/Albumin Nanoparticles for Inhibition of Ferroptosis in Tubular Epithelial Cells during Acute Kidney Injury
}

Liping Deng ${ }^{1}$, Mengqing Xiao ${ }^{1}$, Anshan $\mathrm{Wu}^{2}$, Dong $\mathrm{He}^{3}$, Sanqian Huang ${ }^{4}$, Tanggang Deng ${ }^{5}$, Jiawei $\mathrm{Xiao}^{1}$, Xinyu Chen ${ }^{1}$, Yongbo Peng ${ }^{6 *}, \mathrm{Ke} \mathrm{Cao}^{1 *}$

1 Department of Oncology, Third Xiangya Hospital of Central South University, Changsha, 410013, China

2 Zhuzhou Hospital of Xiangya School of Medicine, Central South University, Zhuzhou, 412007, China

3 Department of Respiration, The Second People's Hospital of Hunan Province, Changsha, 410021, China

4 Department of Pathology, Hunan Cancer Hospital, Changsha, 410000, China

5 Clinical Laboratory, The First Affiliated Hospital of Guangdong Pharmaceutical University, Guangzhou, 510080, China

6 Chongqing Key Laboratory for Pharmaceutical Metabolism Research, The Key Laboratory of Biochemistry and Molecular Pharmacology, College of Pharmacy, Chongqing Medical University, Chongqing, 400016, China

*Correspondence authors: csucaoke@163.com; pengyongbo2000@126.com. 


\section{Supporting Information}

\section{S1}

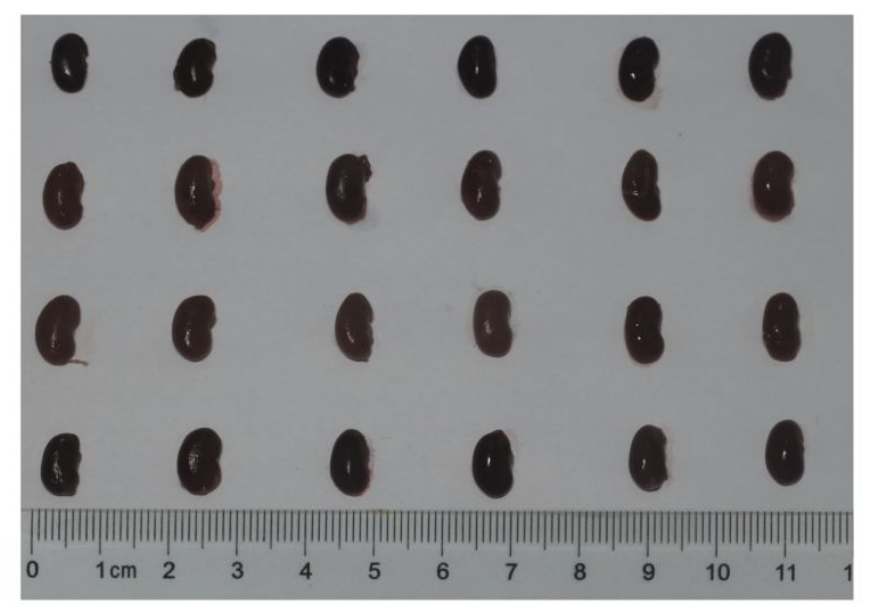

PBS-Saline

PBS-DDP

Se NPs-DDP

SA NPs-DDP

Figure S1. Gross appearance of the congestion area of representative kidneys from each group. 
S2

qRT-PCR of ferroptosis target genes

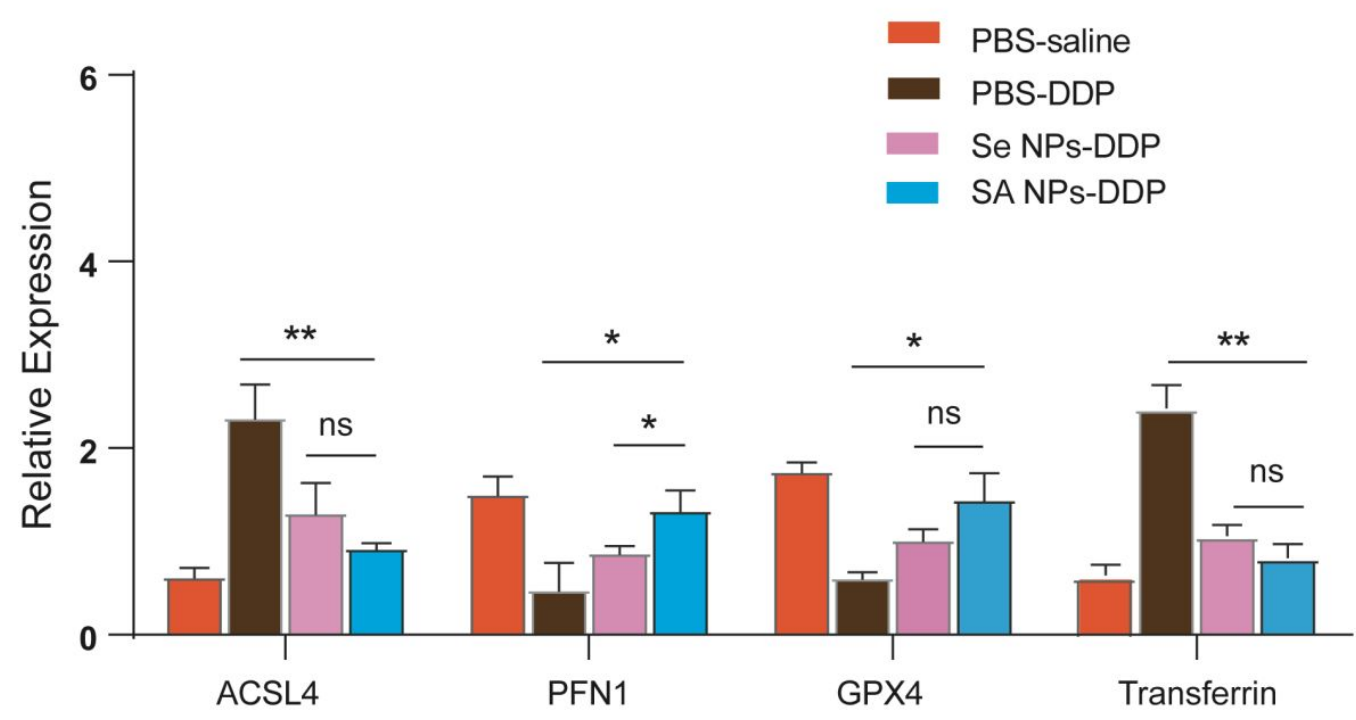

Figure S2. mRNA expression of ferroptosis-associated genes in the renal tubule of the cisplatininduced acutely injured kidney mice ( $\mathrm{n}=3$ per group). 